Check for updates

Cite this: RSC Adv., 2019, 9, 1976

Received 28th November 2018 Accepted 23rd December 2018

DOI: $10.1039 / \mathrm{c} 8 \mathrm{ra09785b}$

rsc.li/rsc-advances

\section{Solid-state AIEnh-circularly polarised luminescence of chiral perylene diimide fluorophores $\uparrow$}

\author{
Ayano Taniguchi, ${ }^{a}$ Daiki Kaji, ${ }^{a}$ Nobuyuki Hara, ${ }^{a}$ Ryosuke Murata, ${ }^{b}$ Shogo Akiyama, ${ }^{a}$ \\ Takunori Harada, ${ }^{\mathrm{c}}$ Atsushi Sudo, ${ }^{a}$ Hiroyuki Nishikawa*b and Yoshitane Imai (iD *a
}

\begin{abstract}
Solid-state organic fluorescent materials are important for the development of electroluminescent sensing devices. Herein, we report that $N, N^{\prime}$-bis((R)-1-phenylethyl)perylene-3,4,9,10-tetracarboxylic diimide $[(R, R)$ BPP] and its antipode [(S,S)-BPP], which contain extended $\pi$-electrons through planar perylenes, emit solidstate aggregation-induced-enhanced (AIEnh) circularly polarised luminescence (CPL) in inorganic $(\mathrm{KBr})$ pellets and organic-polymer-film (PMMA- and myo-IPU-film) states; this CPL is difficult to observe in solution. These chiral perylene fluorophores emit AIEnh-CPL with high dissymmetry factors ( $g_{\mathrm{CPL}}$ ) (up to $\left.2.4 \times 10^{-3}\right)$ and high quantum yields $\left(\Phi_{\mathrm{F}}\right.$, up to 0.43$)$ in the three solid matrices.
\end{abstract}

\section{Introduction}

Because of their potential applications to electroluminescence (EL) and optical-sensing devices, solid-state organic fluorescent materials have attracted much attention. ${ }^{1}$ In particular, optically active organic and inorganic luminophores that emit circularly polarised luminescence (CPL) with high dissymmetry factors $\left(g_{\mathrm{CPL}}\right)$ and high quantum yields $\left(\Phi_{\mathrm{F}}\right)$ have received significant attention in the field of circularly polarised organic light-emitting diodes (CP-OLEDs). ${ }^{2}$ Recently, aggregationinduced-enhanced (AIEnh) CPL has also attracted much interest. However, AIEnh-CPL is almost always emitted from highly concentrated solutions. ${ }^{3}$ Therefore, systems that emit solid-state AIEnh-CPL are highly desired. With the aim of providing a common design approach to novel chiral solid-state AIEnh organic fluorophores, in this study we prepared solidstate AIEnh circularly polarised luminophores by doping chiral $\quad N, N^{\prime}$-bis $((R)$-1-phenylethyl)perylene-3,4,9,10tetracarboxylic diimide $[(R, R)$-BPP $]$ and its antipode $[(S, S)$-BPP $]$ into various types of solid matrix. The two BPPs are chiral bisubstituted perylene fluorophores composed of extended $\pi$ electron planar perylenes. We then examined the AIEnh-CPL emissions from these imbedded BPP systems as AIEnhfluorophore models. ${ }^{4} \mathrm{KBr}$ was used as the solid inorganic

${ }^{a}$ Department of Applied Chemistry, Faculty of Science and Engineering, Kindai University, 3-4-1 Kowakae, Higashi-Osaka, Osaka 577-8502, Japan. E-mail: y-imai@ apch.kindai.ac.jp

${ }^{b}$ Graduate School of Science and Engineering, Ibaraki University, 2-1-1 Bunkyo, Mito, Ibaraki 310-8512, Japan. E-mail: hiroyuki.nishikawa.sci@vc.ibaraki.ac.jp ${ }^{c}$ Department of Integrated Science and Technology, Faculty of Science and Technology, Oita University, Dannoharu 700, Oita City 870-1192, Japan

$\dagger$ Electronic supplementary information (ESI) available. See DOI: 10.1039/c8ra09785b matrix, while poly(methyl methacrylate) (PMMA) and polyurethane (myo-IPU) were chosen as solid organic polymer matrices. ${ }^{5}$ Myo-IPU is derived from naturally occurring myoinositol (a meso-hexose) and characteristically has a higher glass transition temperature $\left(T_{\mathrm{g}} \sim 150{ }^{\circ} \mathrm{C}\right)$ than conventional PMMA $\left(T_{\mathrm{g}} \sim 105^{\circ} \mathrm{C}\right)$.

Herein, we report that $(R, R)$ - and $(S, S)$-BPP emit solid-state AIEnh-CPL with high quantum efficiencies when imbedded in solid inorganic and organic polymer matrices (Fig. 1).

\section{Experimental}

\section{General}

The $\mathrm{KBr}$ and PMMA matrices for solid pellets and films were purchased from JASCO (Tokyo, Japan) and the FUJIFILM Wako Pure Chemical Corp. (Osaka, Japan), respectively. Polyurethane (myo-IPU) was prepared by a previously reported method. ${ }^{5}$ Chloroform $\left(\mathrm{CHCl}_{3}\right)$ used for the determination of solutionstate optical properties was purchased from the FUJIFILM Wako Pure Chemical Corp. (Osaka, Japan).

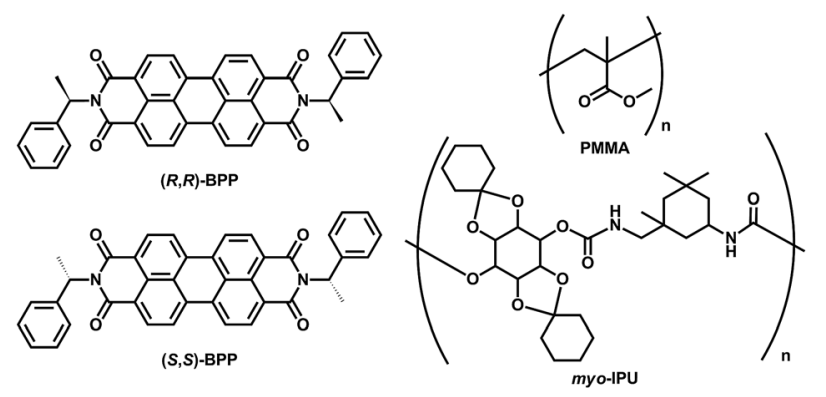

Fig. 1 The chiral perylene fluorophores ((R,R)-BPP and (S,S)-BPP) and organic polymer matrices (PMMA and myo-IPU) used in this study. 
Syntheses of $N, N^{\prime}$-bis $((R)$-1-phenylethyl)perylene-3,4,9,10tetracarboxylic diimide $[(R, R)-\mathrm{BPP}]$ and $(S, S)$-BPP

$(R, R)$-BPP and $(S, S)$-BPP were prepared with reference to a previously reported method. ${ }^{4}$ A mixture of 3,4,9,10-perylenetetracarboxylic dianhydride (430.0 mg, $1.1 \mathrm{mmol}),(R)-1-$ phenylethylamine $(0.3 \mathrm{~mL}, 2.4 \mathrm{mmol})$, and zinc acetate dihydrate $(240.1 \mathrm{mg}, 1.1 \mathrm{mmol})$ in quinolone $(20 \mathrm{~mL})$ was heated with stirring at $180{ }^{\circ} \mathrm{C}$ under nitrogen for $4 \mathrm{~h}$. After cooling to room temperature, the reaction mixture was treated with $2 \mathrm{~N}$ $\mathrm{HCl}$ and stirred at room temperature overnight. The resulting dark-red precipitate was collected by filtration, washed thoroughly with water and methanol $(\mathrm{MeOH})$, and dried in vacuo. The crude product was purified by silica-gel column chromatography using $\mathrm{CHCl}_{3} /$ EtOAc $(20: 1, \mathrm{v} / \mathrm{v})$ as the eluent, to give $523.2 \mathrm{mg}$ (79\% yield) of $(R, R)$-BPP as a deep-red solid. The $S$ enantiomer, namely $(S, S)$-BPP, was synthesised from $(S)$-1phenylethylamine by the same procedure to provide a deep-red solid (76\% yield): ${ }^{1} \mathrm{H}$ NMR for $(R, R)$-BPP $\left(\mathrm{CDCl}_{3} 500 \mathrm{MHz}\right) \delta 2.06$ $(\mathrm{d}, J=7.5 \mathrm{~Hz}, 3 \mathrm{H}), 6.60$ (q, $J=7.5 \mathrm{~Hz}, 2 \mathrm{H}), 7.29$ (t, $J=7.5 \mathrm{~Hz}$, $2 \mathrm{H}), 7.38(\mathrm{t}, J=7.5 \mathrm{~Hz}, 4 \mathrm{H}), 7.57$ (d, $J=7.5 \mathrm{~Hz}, 4 \mathrm{H}), 8.58$ (d, $J=$ $8.0 \mathrm{~Hz}, 4 \mathrm{H}), 8.66(\mathrm{~d}, J=8.0 \mathrm{~Hz}, 4 \mathrm{H}) ;{ }^{13} \mathrm{C} \mathrm{NMR}\left(\mathrm{CDCl}_{3} 125 \mathrm{MHz}\right)$ $\delta 16.3,50.5,122.9,123.5,126.1,127.2,127.5,128.2,129.2,131.3$, 134.3, 140.5, 163.3 .

\section{Unpolarised photoluminescence (PL) quantum yields}

Samples were dispersed in $\mathrm{KBr}$ pellets in the following way. Microcrystalline powdered samples $(10 \mu \mathrm{g})$ were finely ground with $120 \mathrm{mg}$ of $\mathrm{KBr}$, and the mixed powder was compressed at $80 \mathrm{MPa} \mathrm{cm}{ }^{-2}$ under vacuum for $25 \mathrm{~min}$ to provide a $10 \mathrm{~mm}$ diameter, $0.7 \mathrm{~mm}$-thick transparent disk. Samples dispersed in PMMA or myo-IPU films were prepared using PMMA or myoIPU $\left(1.0 \times 10^{-2} \mathrm{M}\right)$ in a spin coater at $3000 \mathrm{rpm}$ (Opticoat MSA100, Mikasa, Tokyo, Japan).

Absolute unpolarised photoluminescence (PL) quantum yields $\left(\Phi_{\mathrm{F}}\right)$ of the KBr-pellet-dispersed states, the PMMA- or myoIPU-film-dispersed states, and $\mathrm{CHCl}_{3}$ solutions were determined using a Hamamatsu Photonics C9920-02 absolute quantum yield spectrometer (Hamamatsu, Japan) in air at room temperature, at excitation wavelengths of $480 \mathrm{~nm}(\mathrm{KBr})$ and $450 \mathrm{~nm}$ (polymer film), respectively, and at $450 \mathrm{~nm}$ for the $\mathrm{CHCl}_{3}$ solution. A $10 \mathrm{~mm}$ path length was used for solutionphase spectroscopy.

\section{Solid-state PL and CPL spectroscopy}

Solid-state PL and CPL spectra of the KBr-pelleted samples were acquired by comprehensive chiroptical spectrophotometry (CCS, JASCO, Tokyo, Japan). ${ }^{6}$ CPL spectra were recorded with a slit width of $33 \mathrm{~mm}$ and a $10 \mathrm{~nm}$ spectral bandwidth for the excitation and emission monochromators. The PL and CPL spectra of the PMMA or myo-IPU films and the $\mathrm{CHCl}_{3}$ solutions were acquired using a JASCO CPL-300 spectrofluoropolarimeter (Tokyo, Japan) at room temperature, at a scattering angle of $0^{\circ}$ upon excitation with unpolarised, monochromated incident light with a $10 \mathrm{~nm}$ bandwidth. An excitation wavelength of $480 \mathrm{~nm}$ was used for the KBr-pelleted samples, while $450 \mathrm{~nm}$ was used for the PMMA or myo-IPU films, and for the $\mathrm{CHCl}_{3}$ solutions. A $10 \mathrm{~mm}$ path length was used for solution-phase spectroscopy.

\section{Circular dichroism (CD) and UV-Vis absorption spectroscopy}

Circular dichroism (CD) and UV-Vis absorption spectra of the $\mathrm{KBr}$-pelleted samples were acquired by CCS at room temperature. ${ }^{6}$ The CD and UV-Vis absorption spectra of PMMA- or myoIPU-film-dispersed states and $\mathrm{CHCl}_{3}$ solutions were recorded with a JASCO J-820 spectropolarimeter at room temperature. The KBr-pelleted and PMMA (or myo-IPU) films were prepared as described for the samples used for solid-state PL and CPL spectroscopy. A $1 \mathrm{~mm}$ path length was used for solution-phase spectroscopy.

\section{Results and discussion}

Prior to elucidating the solid-state chiroptical properties of $(R, R)$-BPP and $(S, S)$-BPP, we first investigated their PL, CPL, and $\mathrm{CD}$ spectral characteristics in $\mathrm{CHCl}_{3}$ solution, at room temperature. To determine whether or not the obtained results are due to artefacts introduced by $(R, R)$-BPP and other factors, the CPL spectrum of $(S, S)$-BPP was also acquired. The CPL and PL spectra of $(R, R)$-BPP and $(S, S)$-BPP are shown in Fig. $2((R, R)$-BPP in blue; $(S, S)$-BPP in red). When dissolved in $\mathrm{CHCl}_{3}\left(1.0 \times 10^{-4}\right.$ M), $(R, R)$-BPP exhibited PL at 549, 576, $623 \mathrm{~nm}$ with an absolute photoluminescence quantum yield $\left(\Phi_{\mathrm{F}}\right)$ of 0.76 . Unfortunately, meaningful CPL spectra of chiral BPP could not be acquired under these conditions.

The CD and UV-Vis absorption spectra of $(R, R)$-BPP and $(S, S)$ BPP in $\mathrm{CHCl}_{3}$ are shown in Fig. 3; as expected, the CD spectra are almost mirror images.

Absorption bands at 459, 490, and $527 \mathrm{~nm}$ that originate from $\pi-\pi^{*}$ transitions in the perylene units are commonly observed, irrespective of the chirality of the sample. As same as CPL properties in the solution-dissolved state, these corresponding CD bands could not be mainly observed.

For the chiral BPPs to emit CPL, we turned to solid-state AIEnh-CPL spectroscopy. The solid-state PL and CPL spectra

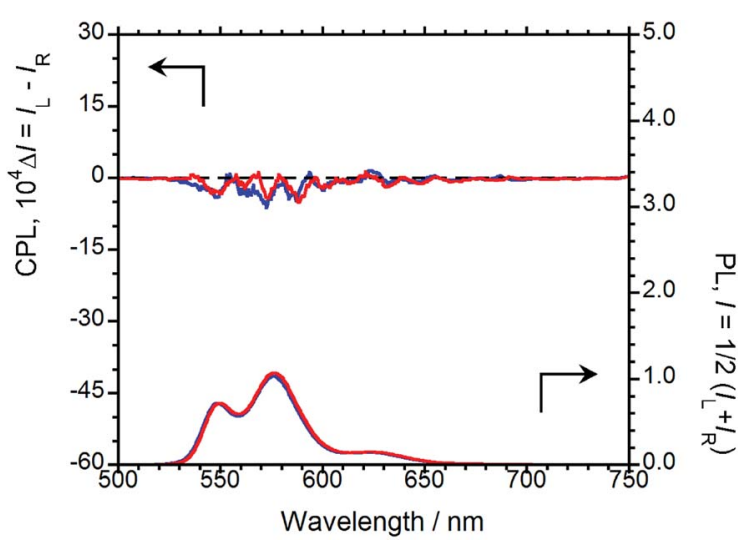

Fig. $2 \mathrm{CPL}$ (upper) and PL (lower) spectra of $(R, R)$ - BPP (blue) and $(S, S)$ $\mathrm{BPP}(\mathrm{red})$ in $\mathrm{CHCl}_{3}\left(1.0 \times 10^{-4} \mathrm{M}\right)$. 


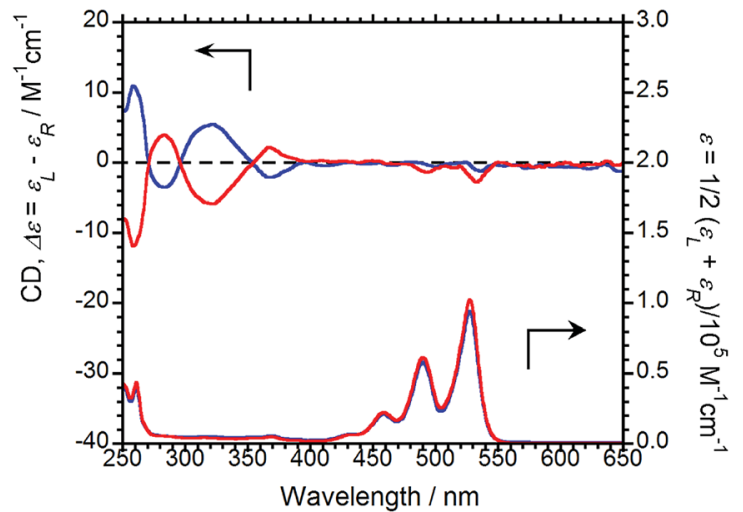

Fig. $3 \mathrm{CD}$ (upper) and UV-Vis absorption (lower) spectra of $(R, R)$-BPP (blue) and (S,S)-BPP (red) in $\mathrm{CHCl}_{3}\left(1.0 \times 10^{-4} \mathrm{M}\right)$.

of $(R, R)$-BPP were acquired in the inorganic $\mathrm{KBr}$ matrix, the results of which are shown in Fig. 4. In contrast to the results in $\mathrm{CHCl}_{3}$ solution, solid-state CPL was observed from the $\mathrm{KBr}-$ pelleted sample; $(R, R)$-BPP in the KBr pellet exhibited a $\lambda_{\mathrm{CPL}}$ of $657 \mathrm{~nm}$ with a corresponding $\Phi_{\mathrm{F}}$ of 0.09 . When the spectrum of the KBr-pellet-dispersed state is compared with that of the $\mathrm{CHCl}_{3}$ solution, quite different $\lambda_{\mathrm{PL}}$ values are observed $(576 \mathrm{~nm}$ for $\mathrm{CHCl}_{3}$ and $632 \mathrm{~nm}$ for $\mathrm{KBr}$ ). The $\lambda_{\mathrm{PL}}$ of the $\mathrm{KBr}$ pellet suggests that CPL from this state is AIEnh CPL derived from the aggregation of $(R, R)$-BPP in the solid state. Unfortunately, the value of $\Phi_{\mathrm{F}}$ in the $\mathrm{KBr}$-dispersed state was smaller than that in $\mathrm{CHCl}_{3}$ solution, which is ascribable to fluorescence quenching under high-density conditions in the solid compared to the lowconcentration conditions in solution. In addition, the external heavy-atom (Br) effect may also be partially responsible.

A negative (-) CPL spectrum was observed for $(R, R)$-BPP in the solid state. To determine whether or not these results are due to artefacts introduced by $(R, R)$-BPP and the KBr matrix, the CPL spectrum of $(S, S)$-BPP was acquired (Fig. 4). $(R, R)$-BPP and $(S, S)$-BPP in $\mathrm{KBr}$ matrices exhibit almost mirror-image CPL spectra. The magnitude of the circular polarisation in the excited state is defined as $g_{\mathrm{CPL}}=\Delta I / I=\left(I_{\mathrm{L}}-I_{\mathrm{R}}\right) /\left(I_{\mathrm{L}}+I_{\mathrm{R}}\right)$, where $I_{\mathrm{L}}$ and $I_{\mathrm{R}}$ are the output signals for left and right circularly

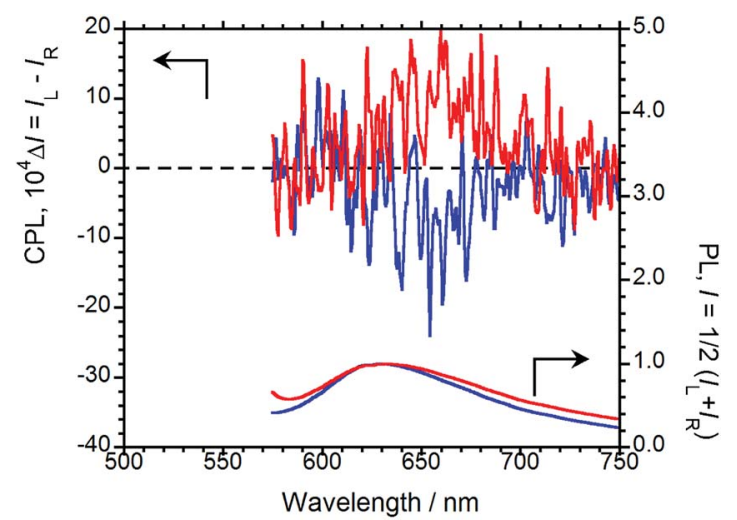

Fig. 4 CPL (upper) and PL (lower) spectra of $(R, R)$ - BPP (blue) and $(S, S)$ $\mathrm{BPP}(\mathrm{red})$ in $\mathrm{KBr}$-pellet-dispersed states. polarised light under unpolarised photoexcitation conditions. The CPL efficiency, $\left|g_{\mathrm{CPL}}\right|$, of BPP in the $\mathrm{KBr}$ pellet is of the order of $10^{-3}$, while the $\left|g_{\mathrm{CPL}}\right|$ of $\mathbf{B P P}$ is $\sim 2.0 \times 10^{-3}$ in the $\mathrm{KBr}-$ dispersed state.

To examine molecular chirality in the ground state, the $\mathrm{CD}$ and UV-Vis absorption spectra of $(R, R)$-BPP and $(S, S)$-BPP in their $\mathrm{KBr}$-pellet-dispersed states were acquired, the results of which are shown in Fig. 5.

Several UV-Vis bands between 400 and $650 \mathrm{~nm}$, which are characteristic of $\pi-\pi *$ transitions in the perylene groups of BPP, can be observed. In this state, the Cotton CD bands of $(R, R)$-BPP and $(S, S)$-BPP, are almost mirror images and are due to $\pi-\pi^{*}$ transitions, as evidenced by their UV-Vis spectra. The CD spectra of $(R, R)$-BPP and $(S, S)$-BPP are almost unaffected by KBrpellet dispersion. Interestingly, the $\mathrm{CD}$ spectra of the $\mathrm{KBr}$ dispersed states are quite different to those of the $\mathrm{CHCl}_{3}$ solutions are similar to those of the previously reported nanoscale aggregates in the solution-dispersed state. ${ }^{4 g}$ We conclude that the $\mathrm{CD}$ spectra of the $\mathrm{KBr}$-dispersed states also result from intermolecular interactions between the perylene units of multiple aggregated BPP molecules within the $\mathrm{KBr}$ pellets. To quantitatively evaluate the $\mathrm{CD}$ amplitude in the ground state, we evaluated the anisotropy factor. The magnitude of the circular polarisation in the ground state is defined as $g_{\mathrm{CD}}=\left(\mathrm{Abs}_{\mathrm{L}}-\mathrm{Abs}_{\mathrm{R}}\right) /\left[\left(\mathrm{Abs}_{\mathrm{L}}+\mathrm{Abs}_{\mathrm{R}}\right) / 2\right]$, where $\mathrm{Abs}_{\mathrm{L}}$ and $\mathrm{Abs}_{\mathrm{R}}$ are the absorbances of the left and right circularly polarised light, respectively. The value of $\left|g_{\mathrm{CD}}\right|$ at the first Cotton CD band of BPP in $\mathrm{KBr}$ was determined to be $\sim 5.4 \times 10^{-4}$ at $561 \mathrm{~nm}$. The observed $\mathrm{CD}$ results in $\mathrm{KBr}$ support the CPL observations mentioned above. Interestingly, a comparison of these anisotropy factors, i.e., the $\left|g_{\mathrm{CPL}}\right|$ and $\left|g_{\mathrm{CD}}\right|$ values, reveals that the $\left|g_{\mathrm{CPL}}\right|$ is larger than the corresponding $\left|g_{\mathrm{CD}}\right|$ value, indicating that, although the perylene units are stacked and twisted in the ground state, ${ }^{4 f}$ the twists increase in the photoexcited state, as confirmed by the intense excimer CPL bands.

In order to emit stronger CPL, we next examined CPL from organic polymer-film states. As an organic polymer matrix, poly(methyl methacrylate) (PMMA) and polyurethane (myo-IPU) derived from naturally occurring myo-inositol were used. The CPL and PL spectra of $(R, R)$-BPP and $(S, S)$-BPP dispersed in

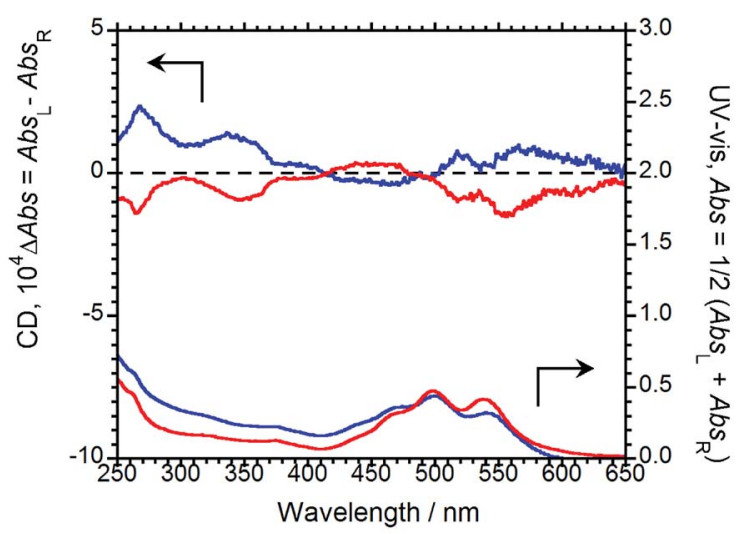

Fig. $5 \mathrm{CD}$ (upper) and UV-Vis (lower) absorption spectra of $(R, R)$-BPP (blue) and (S,S)-BPP (red) in KBr-pellet-dispersed states. 
PMMA and myo-IPU films are shown in Fig. 6(a) and (b), respectively.

$(R, R)$-BPP in PMMA emitted at $654 \mathrm{~nm}\left(\lambda_{\mathrm{CPL}}\right)$ with a $\Phi_{\mathrm{F}}$ of 0.40 , while in myo-IPU, it emitted at $637 \mathrm{~nm}\left(\lambda_{\mathrm{CPL}}\right)$ with a $\Phi_{\mathrm{F}}$ of 0.43. As expected, $(R, R)$-BPP exhibits solid-state AIEnh-CPL in both polymers, with several regressional phonon sidebands originating from the lowest $\pi-\pi^{*}$ transitions between perylene groups observed. The CPL spectra of $(R, R)$-BPP in PMMA and myo-IPU films mostly exhibited negative (-) CPL bands (Fig. 6(a) and (b), respectively). As expected, $(S, S)$-BPP exhibited positive (+) CPL spectra in these polymers (Fig. 6(a) and (b)). Although the CPL spectral shapes of $(R, R)$-BPP and $(S, S)$-BPP in the KBr-pellet and PMMA- and myo-IPU-film states are nearly mirror images, subtle differences in the shapes of the CPL signals are noted. These differences may be due to the effect of humidity in the pellets and films during the preparation of the pellet and film samples. The $\left|g_{\text {CPL }}\right|$ values of BPP in the PMMA and myo-IPU states are $\sim 2.4 \times 10^{-3}$ and $\sim 1.3 \times 10^{-3}$, respectively, which are similar to that observed in the $\mathrm{KBr}$ state $(\sim 2.0$ $\left.\times 10^{-3}\right)$.

As expected, despite the CPL wavelengths of $(R, R)$-BPP in the $\mathrm{KBr}$ and polymer states being similar, the values of $\Phi_{\mathrm{F}}$ in these polymers are significantly higher than that observed for the $\mathrm{KBr}$ state (0.09). We assume that these approximately five-timeshigher values are due to the suppression of thermal deactivation modes and effective packing arrangements because the molecular geometry of the chiral BPP is effectively and suitably fixed by the surrounding solid polymer matrix without quenching.

The CD and UV-Vis absorption spectra of $(R, R)$-BPP and $(S, S)$ BPP in the PMMA and myo-IPU films are shown in Fig. 7(a) and (b), respectively; the CD spectra in these polymers are similar in

(a)

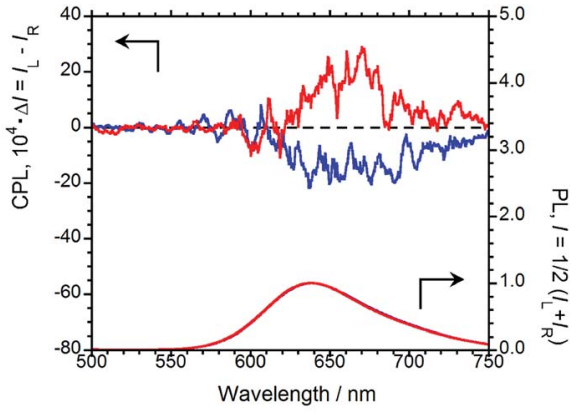

(b)

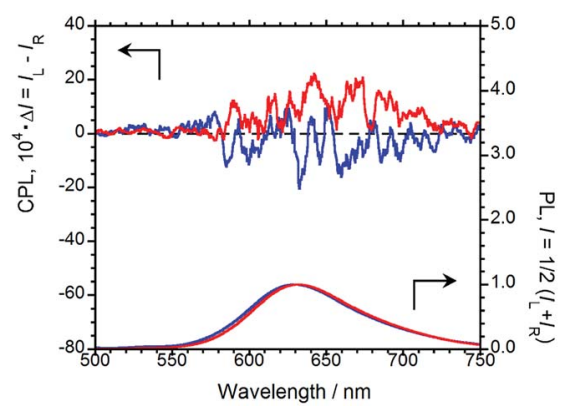

Fig. 6 CPL (upper) and PL (lower) spectra of $(R, R)$-BPP (blue) and $(S, S)$ BPP (red) in (a) PMMA-film- and (b) myo-IPU-film-dispersed states. (a)

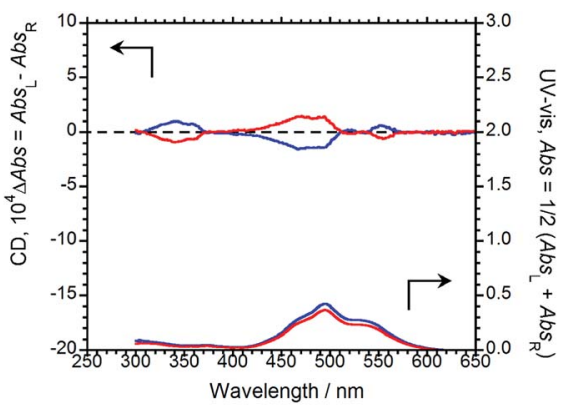

(b)

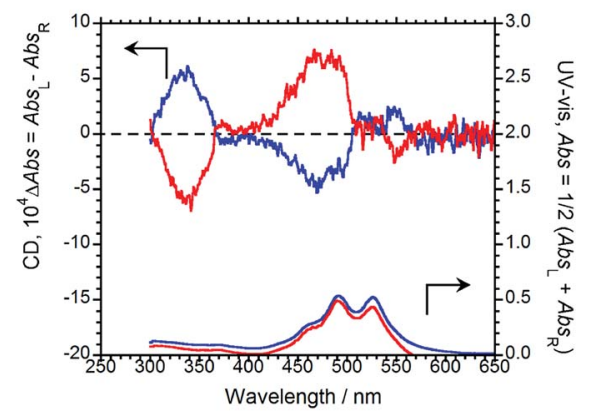

Fig. $7 \mathrm{CD}$ (upper) and UV-Vis absorption (lower) spectra of $(R, R)$-BPP (blue) and (S,S)-BPP (red) in (a) PMMA-film- and (b) myo-IPU-filmdispersed states.

their long-wavelength tails and are similar to those of the $\mathrm{KBr}$ dispersed states. These results suggest that the CD spectra of the polymer-dispersed states are essentially due to intermolecular interactions between multiple molecules rather than intramolecular interactions within individual BPP molecules. The characteristic UV peaks originating from $\pi-\pi^{*}$ transitions of the perylene groups are commonly observed between 400 and $600 \mathrm{~nm}$. The CD spectra of $(R, R)$-BPP and $(S, S)$-BPP in both matrices are almost mirror images, as expected. The signs of the first Cotton CD bands of $(R, R)$-BPP, at $553 \mathrm{~nm}$ in PMMA and at $551 \mathrm{~nm}$ in myo-IPU, are both positive $(+)$, while the CD signs of the corresponding bands for $(S, S)$-BPP in PMMS and myo-IPU are both negative $(-)$. The $\left|g_{\mathrm{CD}}\right|$ value at the first Cotton CD bands are $\sim 3.1 \times 10^{-4}$ and $\sim 2.9 \times 10^{-4}$ for the PMMA- and myoIPU-dispersed states, respectively. BPP in these matrices exhibit $\left|g_{\mathrm{CD}}\right|$ values of similar magnitude.

As was observed in the $\mathrm{KBr}$ matrix, the $\left|g_{\mathrm{CPL}}\right|$ values are higher than the corresponding $\left|g_{\mathrm{CD}}\right|$ values in the polymer films. This shows that intermolecular twists between perylene units in the ground state increase upon photo-excitation, as evidenced by the intense excimer CPL bands in spectra of the polymer-matrix imbedded samples.

\section{Conclusions}

Chiral $(R, R)$ - and $(S, S)$ - $N, N^{\prime}$-bis(1-phenylethyl)perylene-3,4,9,10tetracarboxylic diimide (BPP) dispersed in solid $\mathrm{KBr}$ and PMMA (or myo-IPU) matrices exhibited solid-state AIEnh-CPL with high $\Phi_{\mathrm{F}}$ values at room temperature. The value of $\Phi_{\mathrm{F}}$ from the PMMA $\left(\Phi_{\mathrm{F}} 0.40\right)$ and myo-IPU matrices $\left(\Phi_{\mathrm{F}} 0.43\right)$ are significantly higher than that observed from the $\mathrm{KBr}$ matrix $\left(\Phi_{\mathrm{F}}\right.$ 
0.09). The present knowledge provides guidance for the design of novel solid chiral organic fluorophoric systems that emit solid-state AIEnh-CPL.

\section{Conflicts of interest}

There are no conflicts to declare.

\section{Acknowledgements}

This study was supported by a Grant-in-Aid for Scientific Research (no. 18K05094) from MEXT/Japan Society for the Promotion of Science, the MEXT-Supported Program for the Strategic Research Foundation at Private Universities (20142018), and a research grant from the International Polyurethane Technology Foundation.

\section{Notes and references}

1 (a) S. R. Forrest, Chem. Rev., 1997, 97, 1793-1896; (b) M. Glasbeek and H. Zhang, Chem. Rev., 2004, 104, 19291954; (c) A. Mishra, R. K. Behera, P. K. Behera, B. K. Mishra and G. B. Behera, Chem. Rev., 2000, 100, 1973-2011; (d) T. Baumgartner and R. Reau, Chem. Rev., 2006, 106, 46814727; (e) P. G. Coble, Chem. Rev., 2007, 107, 402-418; (f) T. P. I. Saragi, T. Spehr, A. Siebert, T. Fuhrmann-Lieker and J. Salbeck, Chem. Rev., 2007, 107, 1011-1065; (g) S. C. Lo and P. L. Burn, Chem. Rev., 2007, 107, 1097-1116; (h) K. Walzer, B. Maennig, M. Pfeiffer and K. Leo, Chem. Rev., 2007, 107, 1233-1271; (i) J. Grate, Chem. Rev., 2008, 108, 726-745; (j) F. Jakle, Chem. Rev., 2010, 110, 3985-4022; (k) V. K. Rai, R. Srivastava and M. N. Kamalasanan, J. Lumin., 2010, 130, 249-253; (l) J. Huang, B. Xu, M. K. Lam, K. W. Cheah, H. C. Chen and J. H. Su, Dyes Pigm., 2011, 89, 155-161; ( $m$ ) V. M. Agranovich, Y. N. Gartstein and M. Litinskaya, Chem. Rev., 2011, 111, 5179-5214; (n) J. E. Kwon and S. Y. Park, Adv. Mater., 2011, 23, 3615-3642; (o) T. H. Tran-Thi, R. Dagnelie, S. Crunaireza and L. Nicole, Chem. Soc. Rev., 2011, 40, 621-639: and references cited therein.

2 (a) K. E. S. Phillips, T. J. Katz, S. Jockusch, A. J. Lovinger and N. J. Turro, J. Am. Chem. Soc., 2001, 123, 11899-11907; (b) J. E. Field, G. Müller, J. P. Riehl and D. Venkataraman, $J$. Am. Chem. Soc., 2003, 125, 11808-11809; (c) H. Maeda, Y. Bando, K. Shimomura, I. Yamada, M. Naito, K. Nobusawa, H. Tsumatori and T. Kawai, J. Am. Chem. Soc., 2011, 133, 9266-9269; (d) R. Tempelaar, A. Stradomska, J. Knoester and F. C. Spano, J. Phys. Chem. B, 2011, 115, 10592-10603; (e) Y. Nakano and M. Fujiki, Macromolecules, 2011, 44, 7511-7519; $(f)$ N. Nishiguchi, T. Kinuta, Y. Nakano, T. Harada, N. Tajima, T. Sato, M. Fujiki, R. Kuroda, Y. Matsubara and Y. Imai, Chem.-Asian J., 2011, 6, 1092-1098; (g) J. Liu, H. Su, L. Meng, Y. Zhao, C. Deng, J. C. Y. Ng, P. Lu, M. Faisal, J. W. Y. Lam, X. Huang, H. Wu, K. S. Wong and B. Z. Tang, Chem. Sci., 2012, 3, 2737-2747; (h) Y. Sawada, S. Furumi, A. Takai, M. Takeuchi, K. Noguchi and K. Tanaka, J. Am. Chem. Soc., 2012, 134, 4080-4083; (i)
H. Oyama, K. Nakano, T. Harada, R. Kuroda, M. Naito, K. Nobusawa and K. Nozaki, Org. Lett., 2013, 15, 2104-2107; (j) T. Shiraki, Y. Tsuchiya, T. Noguchi, S.-I. Tamaru, N. Suzuki, M. Taguchi, M. Fujiki and S. Shinkai, Chem.Asian J., 2014, 9, 218-222; (k) X. Jiang, X. Liu, Y. Jiang, Y. Quan, Y. Cheng and C. Zhu, Macromol. Chem. Phys., 2014, 215, 358-364; (l) Y. Morisaki, M. Gon, T. Sasamori, N. Tokitoh and Y. Chujo, J. Am. Chem. Soc., 2014, 136, 3350-3353; ( $m$ ) S. Abbate, G. Longhi, F. Lebon, E. Castiglioni, S. Superchi, L. Pisani, F. Fontana, F. Torricelli, T. Caronna, C. Villani, R. Sabia, M. Tommasini, A. Lucotti, D. Mendola, A. Mele and D. A. Lightner, J. Phys. Chem. C, 2014, 118, 1682-1695; (n) K. Watanabe, Y. Koyama, N. Suzuki, M. Fujiki and T. Nakano, Polym. Chem., 2014, 5, 712-717; (o) E. M. Sánchez-Carnerero, A. R. Agarrabeitia, F. Moreno, B. L. Maroto, G. Muller, M. J. Ortiz and S. de la Moya, Chem.-Eur. J., 2015, 21, 13488-13500; (p) Y. Uchida, T. Hirose, T. Nakashima, T. Kawai and K. Matsuda, Org. Lett., 2016, 18, 2118-2121; (q) M. Shimada, Y. Yamanoi, T. Ohto, S. T. Pham, R. Yamada, H. Tada, K. Omoto, S. Tashiro, M. Shinoya, M. Hatton, K. Jimura, S. Hayashi, H. Koike, M. Iwamura, K. Nozaki and H. Nishihara, J. Am. Chem. Soc., 2017, 139, 11214-11221; (r) H. Tanaka, Y. Inoue and T. Mori, ChemphotoChem, 2018, 2, 1-18; (s) K. Takaishi, R. Takehana and T. Ema, Chem. Commun., 2018, 54, 14491452, and references cited therein.

3 (a) H.-Z. Tang, M. Fujiki and M. Motonaga, Polymer, 2002, 43, 6213-6220; (b) S. Haraguchi, M. Numata, C. Li, Y. Kawagoe, M. Fujiki and Y. Nakano, New J. Chem., 2010, 34, 637-647; (c) K. Watanabe, T. Sakamoto, M. Taguchi, M. Fujiki and T. Nakano, Chem. Commun., 2011, 47, 10996-10998; (d) K. Okano, M. Taguchi, M. Fujiki and T. Yamashita, Angew. Chem., Int. Ed., 2011, 50, 12474-12477; (e) Y. Nakano, F. Ichiyanagi, M. Naito, Y. G. Yang and M. Fujiki, Chem. Commun., 2012, 48, 6636-6639; (f) M. Fujiki, A. J. Jalilah, N. Suzuki, M. Taguchi, W. Zhang, M. M. Abdellatif and K. Nomura, RSC Adv., 2012, 2, 6663-6671; (g) M. Fujiki, Y. Kawagoe, Y. Nakano and A. Nakao, Molecules, 2013, 18, 7035-7057; (h) H. Maeda and Y. Bando, Pure Appl. Chem., 2013, 85, 1967-1978; (i) T. Shiraki, Y. Tsuchiya, T. Noguchi, S.-i. Tamaru, N. Suzuki, M. Taguchi, M. Fujiki and S. Shinkai, Chem.-Asian J., 2014, 9, 218-222; (j) K. Watanabe, Y. Koyama, N. Suzuki, M. Fujiki and T. Nakano, Polym. Chem., 2014, 5, 712-717; (k) M. Fujiki, Y. Donguri, Y. Zhao, A. Nakao, N. Suzuki, K. Yoshida and W. Zhang, Polym. Chem., 2015, 6, 1627-1638; (l) M. Fujiki and S. Yoshimoto, Mater. Chem. Front., 2017, 1, 1773-1785; (m) C. Zhang, L. Meng, H.-Y. Lu and C.-F. Chen, RSC Adv., 2018, 8, 1014-1021.

4 (a) E. K. Todd, S. Wang, X. Wan and Z. Y. Wang, Tetrahedron Lett., 2005, 46, 587-590; (b) B. M. Rumyantsev, V. I. Berendyaev, A. Y. Tsegelskaya, T. S. Zhuravleva and I. V. Klimenko, Synth. Met., 2005, 152, 85-88; (c) S. Amiralaei, D. Uzun and H. Icil, Photochem. Photobiol. Sci., 2008, 7, 936-947; (d) A. Pucci, F. Donati, S. Nazzi, G. U. Barretta, G. Pescitelli, L. D. Bari and G. Ruggeri, React. 
Funct. Polym., 2010, 70, 951-960; (e) B. M. Rumyantsev, V. I. Berendyaev and D. V. Pebalk, J. Phys. Chem. B, 2014, 8, 205-211; $(f)$ X. Shang, I. Song, Hi. Ohtsu, Y. H. Lee, T. Zhao, T. Kojima, J. H. Jung, M. Kawano and J. H. Oh, Adv. Mater., 2017, 29, 1605828; $(g)$ X. Shang, I. Song, H. Ohtsu, J. Tong, H. Zhang and J. H. Oh, Sci. Rep., 2017, 7, 5508.
5 T. Amako, K. Nakabayashi, A. Sudo, M. Fujiki and Y. Imai, Org. Biomol. Chem., 2015, 13, 2913-2917.

6 T. Harada, H. Hayakawa, M. Watanabe and M. Takamoto, Rev. Sci. Instrum., 2016, 87, 075102. 